\title{
Relative validity of an FFQ for assessing dietary fluoride intakes of infants and young children living in lowa
}

\author{
Scott J Rankin ${ }^{1, *}$, Steven M Levy², John J Warren², Julie E Gilmore ${ }^{3}$ \\ and Barbara Broffitt ${ }^{2}$ \\ ${ }^{1}$ Public Health Dentist, US Army, 2050 Worth RD, MCDS, Fort Sam Houston, TX 78249, USA: ${ }^{2}$ University of \\ lowa College of Dentistry, Department of Preventive and Community Dentistry, lowa City, IA, USA: ${ }^{3}$ University of \\ lowa Institute for Clinical \& Translational Science, lowa City, IA, USA
}

Submitted 6 July 2010: Accepted 9 February 2011: First published online 31 March 2011

\begin{abstract}
Objective: To determine the relative validity of a quantitative FFQ in assessing dietary fluoride intakes using $3 \mathrm{~d}$ food and beverage diaries for reference.

Design: Parents were asked to complete questionnaires for the preceding week and diaries for $3 \mathrm{~d}$ for their children. Fluoride intakes were estimated from 'selected' foods and beverages for questionnaires and from 'all foods and beverages' for diaries. Data collected at 6, 9, 12, 16, 20, 24, 36, 48 and 60 months were analysed cross-sectionally.

Setting: A 3d food and beverage diary and an FFQ collected through mail from children living in the state of Iowa.

Subjects: Children from the Iowa Fluoride Study whose parents completed both an FFQ and a $3 \mathrm{~d}$ food and beverage diary at each analysed time point.

Results: Correlations between daily mean dietary fluoride intake estimated from questionnaires and diaries range from 0.90 to 0.65 .

Conclusions: A quantitative FFQ can provide relative estimates of dietary fluoride intake.
\end{abstract}

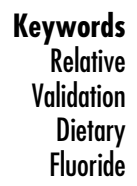

When seeking to assess dietary intakes at the population level, investigators have to consider many factors before choosing the type of dietary assessment tool. Factors to be considered include how detailed, population-specific and expensive the tool will be for the purpose of the study ${ }^{(1)}$. Although multiple $24 \mathrm{~h}$ recalls and diet diaries can provide valid assessments of an individual's usual intake and a level of detail not possible with other approaches, they can be very time-consuming and expensive to administer, complete and analyse. The level of detail provided by these approaches may not be necessary or practical for studies with large sample sizes ${ }^{(2)}$.

When conducting epidemiological studies with large sample sizes, it is often important to use a dietary assessment tool that is not only valid but also easy to use for both the research participant and the researcher, and that also minimizes costs ${ }^{(3)}$. The FFQ was created in an attempt to obtain a self-administered, inexpensive and rapid estimate of usual intake and is usually constructed to emphasize the recording of the intake of one or more nutrients of interest.

Fluoride has long been recognized as an effective agent in preventing dental caries. Constant, low levels of fluoride in the mouth help to prevent caries by promoting remineralization and by helping to inhibit demineralization $^{(4)}$. This low concentration of fluoride in the oral cavity is obtained through direct contact with fluoride agents (i.e. ingesting foods and beverages with fluoride) and also through the secretion of fluoride from the salivary glands as a result of systemic intake ${ }^{(5)}$. The major health concerns of high levels of fluoride intake are increased risk of developing dental and/or skeletal fluorosis ${ }^{(4)}$.

Marshall et $a l^{(2)}$ evaluated the relative validity of a semiquantitative beverage questionnaire in assessing beverage volumes as well as $\mathrm{Ca}$ and vitamin D intakes using $3 \mathrm{~d}$ diet diaries for reference measurements. The Spearman correlations for beverage intake were high for human milk ( $r=0.99$ at 6 months), infant formula $(r=0.84$ at 6 months), cow's milk ( $r=0.86$ at 6 months and $r=0.76$ at 12 months) and juice/juice drinks $(r=0.69$ at 6 months and $r=0.64$ at 12 months). Correlations for $\mathrm{Ca}$ and vitamin $\mathrm{D}$ at 6 months were $r=0.64$ and $0 \cdot 80$, respectively, compared with $r=0.67$ and 0.60 , respectively, at 12 months. The correlation for water intake was not quite as high at 6 months $(r=0.54)$ but increased to $r=0.70$ at 12 months. These findings are important because most dietary fluoride comes from beverages and these results show high correlations for beverage intake estimates between questionnaires and diaries.

Araujo et al. $^{(6)}$ analysed the relative validity of a semiquantitative FFQ (in addition to estimating the calibration factors) designed to estimate the usual food intake 
of adolescents. The study sample consisted of adolescents living in Rio de Janeiro, Brazil. The Pearson correlation coefficients were $r=0 \cdot 17$ for Fe intake, $r=0.33$ for total fat intake, $r=0.46$ for the intake of carbohydrates and $r=0.47$ for $\mathrm{Ca}$ and fibre intakes.

Hacker-Thompson et $a l .{ }^{(7)}$ analysed the validity of two $\mathrm{Ca}$-intake questionnaires. The study sample consisted of community-dwelling women over 18 years of age. The Pearson correlation coefficient for each Ca-intake questionnaire was $r=0.37$ when compared with the $3 \mathrm{~d}$ mean Ca intake from a $3 \mathrm{~d}$ food record.

In 1997, the Institute of Medicine (IOM) released its upper limit (the level below which there is unlikely to be any adverse health effects in healthy people) recommendations for dietary intake of fluoride ${ }^{(8)}$. The IOM recommended these upper limits to be: $0.7 \mathrm{mg} / \mathrm{d}$, $0.9 \mathrm{mg} / \mathrm{d}, 1.3 \mathrm{mg} / \mathrm{d}$ and $2.2 \mathrm{mg} / \mathrm{d}$ for children from birth to 6 months, $7-12$ months, $1-3$ years and $4-8$ years of age, respectively, based on $0 \cdot 1 \mathrm{mg}$ fluoride/ $\mathrm{kg}$ body weight and average weights for children of those ages. The IOM also released Adequate Intakes (AI; used as guides for nutrient intakes for individuals and generally regarded as being compatible with health) for fluoride ingestion ${ }^{(8)}$. The IOM AI values for fluoride are $0 \cdot 01,0 \cdot 5,0 \cdot 7$ and $1 \mathrm{mg}$ fluoride/d from birth to 6 months, 7-12 months, 1-3 years and 4-8 years of age, respectively.

Previous research has attempted to determine the relative validity of FFQ in estimating nutrient as well as food and beverage intakes. To date, no study has determined the relative validity of an FFQ that estimates the intake of dietary fluoride. The purpose of the present research was to determine the relative validity of an FFQ developed as part of the Iowa Fluoride Study (IFS) in assessing fluoride intakes using $3 \mathrm{~d}$ food and beverage diaries for reference measurements.

\section{Methods}

This was a secondary data analysis conducted on data collected as part of the IFS. The overall goal of the IFS has been to investigate dietary (foods and beverages) and non-dietary (dentifrices, dental rinses and gels) fluoride exposures and intakes and their relationships with dental fluorosis and caries in both primary and permanent dentitions. The IFS is a prospective, longitudinal investigation on a cohort recruited at birth from eight Iowa hospitals from March 1992 to February 1995 and has been discussed previously in more detail ${ }^{(5,9-11)}$. The University of Iowa Institutional Review Board approved the study protocol and all participants provided written informed consent. At recruitment, the following initial baseline data were collected from the mothers while they were still in the hospital with their newborns: their age, educational level, family income, number of children in the household, water sources and infant feeding plans.
Questionnaires developed specifically for the IFS and 3d food and beverage diaries were sent to the mothers when the children were 6 weeks of age, and subsequently every 3 months from 3 to 12 months of age, every 4 months from 12 to 48 months of age and every 6 months from 48 to 72 months of age, for a total of eighteen cross-sectional assessments over a period of 6 years ${ }^{(12)}$.

The study questionnaires were mailed to participants at the designated time points (i.e. 6 weeks, 3 and 9 months, etc.) and included items relating to the children's fluoride exposures from dietary and non-dietary sources during the previous time period (i.e. 6-9, 9-12 months, etc.). The questionnaires included specific series of questions regarding the home water supply (such as where did the tap water come from, i.e. is it from a public water supply, from a private well, community well, etc.), whether a water softener was used and what type of filtration system was used, if any. The amount of time the child spent at childcare and/or school and the fluoride levels, origin and filtration status of the water sources of the childcare and/or school were collected as well. A semiquantitative FFQ was a component of the study questionnaire and respondents were asked to list the frequency and quantity of the following food and beverage categories consumed during the $7 \mathrm{~d}$ : (i) water by itself; (ii) water mixed with other foods and beverages; (iii) ready-to-drink beverages not mixed with water; and (iv) ready-to-feed foods not mixed with water. Items 2,3 and 4 were divided into a number of subcategories to assess intake. This tool has been evaluated for validity previously ${ }^{(2)}$.

The FFQ focused on capturing the major dietary categories of fluoride exposures in the children. In most instances, the FFQ did not include complete specific information, such as the brand name of the product consumed, the flavour of the product, the container size, etc. Therefore, the FFQ was more category specific. From 6 to 20 months, the following categories were captured on the FFQ: water by itself, powdered concentrate infant formula (with water), liquid concentrate infant formula (with water), ready-to-feed infant formula, milk, breast milk from pump, ready-to-feed juices/juice drinks (drank as purchased), ready-to-feed juices/juice drinks (diluted), non-juice beverages (drank as purchased), frozen concentrate (mixed as directed), frozen concentrate (diluted), powdered concentrate, ready-to-feed baby food, dry powdered infant cereal or other dry powdered cereal or food, and other food prepared with water. From 24 to 60 months, the following categories were captured on the FFQ: water by itself, milk, ready-to-feed juices/juice drinks (drank as purchased), ready-to-feed juices/juice drinks (diluted), non-juice beverages (drank as purchased), frozen/liquid concentrate (mixed as directed), frozen/liquid concentrate (diluted), powdered concentrate, food prepared only with water (i.e. Jell-O ${ }^{\circledR}$ ), food prepared with some amount of water (i.e. canned soup) and food cooked in water (i.e. rice, pasta, etc.). 


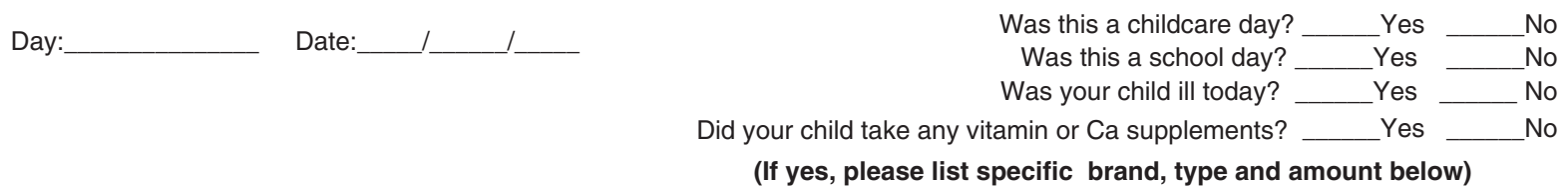

\begin{tabular}{|c|c|c|c|c|c|c|c|}
\hline $\begin{array}{l}\text { Time } \\
\text { (hours) }\end{array}$ & $\begin{array}{l}\text { Place: } \\
\text { H-Home } \\
\text { C-Childcare } \\
\text { S-School } \\
\text { O-Out }\end{array}$ & $\begin{array}{l}\text { Food, beverages and vitamin or } \\
\text { Ca supplements } \\
\text { (complete product description) }\end{array}$ & $\begin{array}{l}\text { Brand name } \\
\text { (for foods and } \\
\text { beverages) } \\
\text { Container size } \\
\text { (for beverages) }\end{array}$ & $\begin{array}{l}\text { Type and/or } \\
\text { preparation: } \\
\text { (e.g. made from } \\
\text { concentrate) }\end{array}$ & $\begin{array}{l}\text { Water from: } \\
\text { H-Home tap } \\
\text { C-Childcare } \\
\text { S-School } \\
\text { O-Out } \\
\text { B-Bottled }\end{array}$ & $\begin{array}{l}\text { Amount ate } \\
\text { and drank }\end{array}$ & $\begin{array}{l}\text { Do not } \\
\text { write } \\
\text { here }\end{array}$ \\
\hline & & & & & & & \\
\hline & & & & & & & \\
\hline & & & & & & & \\
\hline & & & & & & & \\
\hline & & & & & & & \\
\hline & & & & & & & \\
\hline & & & & & & & \\
\hline & & & & & & & \\
\hline & & & & & & & \\
\hline & & & & & & & \\
\hline & & & & & & & \\
\hline & & & & & & & \\
\hline
\end{tabular}

Note: Brand names, complete product description and the manufacturer's container size are needed for all beverages. We will determine fluoride values on the basis of this information. If you have any questions on how to complete this information, please refer to the example page or call us at 1-888-857-7038 if you need further assistance. Thank you.

Fig. 1 Three-day food and beverage diary entry form

There were a total of sixteen items on the questionnaires for participants from 6 to 20 months of age and eleven items for participants from 24 to 60 months of age.

To quantify fluoride intake, each food or beverage category included on the questionnaire was assigned an average fluoride concentration value for the non-water portion of the beverage or food. These average fluoride concentration values were weighted averages of the assays of food and beverages that were tested and found to be the most commonly consumed across the cohort ${ }^{(13-16)}$.

Parents were also asked to complete $3 \mathrm{~d}$ food and beverage diaries (see Fig. 1). The diary format was essentially the same for each of the time points. They were to record all foods and beverages that the child consumed during a selected $72 \mathrm{~h}$, including one weekend day and two weekdays. If the child went to childcare, the parents were requested to ask the daycare provider to record everything the child ate and drank while at daycare. The diaries were primarily completed by the parents. For each day that dietary data were recorded, the parents (or childcare providers) were asked to list the day, date, whether the child was in childcare and whether the child was ill that day or not. The parents (or childcare provider) were instructed to list the time of day the food and/or beverages were consumed, the location where the foods or beverages were consumed (i.e. at home, childcare or other location), the type of food or beverage, brand name and other details such as the size of the container, method of preparation and the amount the child ate and drank.
If water was consumed as a beverage or used during food preparation, the parents were requested to indicate the water source (i.e. tap, bottled, etc.). For mixed dishes such as casseroles, sandwiches, etc., the parent was requested to list each ingredient and its amount. An informational summary was included with each mail to instruct parents on how to record portion sizes. A contact number was included in case the parents had any further questions.

To account for fluoride consumed through water, intakes were evaluated on an individual basis to determine water sources. Non-public water sources and those using filtration systems were analysed for fluoride concentration annually. If water was from a public source and without a home filtration system, fluoride concentrations were obtained from the Iowa State Health Department on a monthly basis. Samples of many readyto-drink beverages and ready-to-eat foods that were reported in the FFQ and $3 \mathrm{~d}$ food and beverage diaries were purchased, analysed at the University of Iowa College of Dentistry laboratory and assigned fluoride levels as part of the IFS. The IFS research team has analysed thousands of food and beverage items for fluoride content. Approximately $10 \%$ of the samples were randomly selected for duplicate analyses. A mean reproducibility rate of $97 \%$ was found ${ }^{(13-16)}$.

The FFQ and $3 \mathrm{~d}$ food and beverage diaries were reviewed and estimates of daily intake were derived. For example, the FFQ asked parents (or childcare providers) to record intakes over the previous $7 \mathrm{~d}$. Individual 
food and beverage entries were multiplied by the amount of fluoride per unit of measurement, then summed up and divided by 7 to determine an estimate of average daily intake. Each food item in the diary was evaluated by registered dietitians to determine its fluoride contribution. Weighted weekly averages of the $3 \mathrm{~d}$ intakes were computed to determine an estimate of average daily intake. To determine the weighted weekly average, the day-specific fluoride totals were extrapolated to five weekdays and two weekend days. These totals were then divided by 7 to derive the average daily intake for a week.

The $3 \mathrm{~d}$ food and beverage diaries provided the ability to capture specific details regarding the dietary fluoride intakes of the children. The diaries included the brand name of the product consumed, the flavour of the product, whether it was sugar-free or regular (if applicable), container size, etc. Therefore, the $3 \mathrm{~d}$ food and beverage diaries were product specific. Using this information, the IFS team was able to assign specific fluoride values from the fluoride assay results to the majority of foods and beverages listed in the diaries.

Responses were systematically reviewed by at least two study members before data entry. Two methods were used to assess the quality of the response to questionnaires and diaries. First, the $3 \mathrm{~d}$ food and beverage diaries were used to cross-check the questionnaires with regard to the types of beverages and foods consumed ${ }^{(5)}$. Second, the reliability of the responses from the FFQ was assessed through telephonic interviews for selected questions $7-10 \mathrm{~d}$ after the initial questionnaires were completed. None of the children had siblings who were also a part of the study. For children aged 6 weeks-36 months, approximately 320 questionnaires (approximately $90 \%$ of unique individuals) were assessed for reliability, with agreement being approximately 96-97\% for questions on water filtration status and approximately $94 \%$ for water consumption $^{(10)}$. Of the questionnaires collected from 36-72-month-old children, approximately 260 questionnaires ( $>80 \%$ unique individuals) were assessed for reliability, with an agreement of approximately $96 \%$ for water filtration status ${ }^{(12)}$.

\section{Statistical metbods}

Non-parametric tests were used for analyses because dietary fluoride intakes were not normally distributed. The Wilcoxon signed-rank test was used to determine the statistical significance of differences between dietary fluoride intakes as recorded in $3 \mathrm{~d}$ food and beverage diaries and the FFQ. Spearman's correlation coefficients were used to compare dietary fluoride intakes from diaries compared with questionnaires.

Data were analysed using the SAS statistical software package version $9 \cdot 1 \cdot 3$ (service pack 4, 2008; SAS Institute Inc., Cary, NC, USA). A $P$ value of $<0.05$ was considered statistically significant.

\section{Results}

Demographic characteristics of the study sample are presented in Table 1. Parents were relatively well educated and of higher socio-economic status (SES). The study sample was predominantly white, similar to the population of Iowa.

The initial sample size of 753 is reflective of the children who completed a $3 \mathrm{~d}$ food and beverage diary and an FFQ at the first time point of collection ( 6 weeks of age). Sample sizes ranged from 376 to 670 at the different ages analysed for the present study. Inclusion in analysis required that both a $3 \mathrm{~d}$ food and beverage diary and an FFQ be present at the analysed age. Response rates were higher at earlier ages and tended to be lower as the age of the study participant increased. Table 2 shows the distributions of estimated daily total fluoride intakes from dietary sources, to include solid foods and beverages, as recorded by the $3 \mathrm{~d}$ food and beverage diary and the FFQ. The results were positively skewed, with means being higher than the medians, and there was substantial variation in the distributions of intakes at all ages (with the SD mostly being almost as large as the means). Maximum intakes tended to be four to five times higher than the means. When evaluating dietary fluoride intakes as reported in the diaries, $25 \%$ of children from birth to 6 months (Table 2) consumed amounts greater than the tolerable upper intake limit (UL) of $0.7 \mathrm{mg} / \mathrm{d}$. Again, using dietary fluoride intakes as reported from the diaries at 12 months of age, $5-10 \%$ of children consumed amounts greater than the tolerable UL of $0.9 \mathrm{mg} / \mathrm{d}$. At 24 and 36 months of age, using intake values reported from the diaries, $<5 \%$ of children consumed amounts of fluoride greater than the tolerable UL of $1.3 \mathrm{mg} / \mathrm{d}$. At 48 and 60 months of age, using intakes reported from the diaries,

Table 1 Demographics of the sample ( $n$ 753)

\begin{tabular}{llr}
\hline Variable & Category & $\%$ \\
\hline Sex & Male & $48 \cdot 5$ \\
& Female & 51.5 \\
Race & White & $97 \cdot 2$ \\
& Other & $2 \cdot 8$ \\
Mother's educational level & Up to high school & $21 \cdot 9$ \\
& Some college & $33 \cdot 9$ \\
Father's educational level & College graduate or more & $44 \cdot 1$ \\
& Up to high school & $26 \cdot 7$ \\
& Some college & $27 \cdot 0$ \\
SES & College graduate or more & $39 \cdot 0$ \\
& Other & $7 \cdot 3$ \\
& Low & 20.5 \\
& Middle & $40 \cdot 1$ \\
& High & $35 \cdot 9$ \\
& Not listed & 3.5 \\
\hline
\end{tabular}

SES, socio-economic status.

*SES was defined from recruitment questionnaires from 1992 to 1995; low SES was defined as family income $<\$$ US 30000 and mother did not have a 4-year college degree; high SES was defined as family income $\geq \$ U S$ 50000 and mother had graduate professional schooling; other combinations were defined as middle SES. 
$<1 \%$ of the children consumed amounts of fluoride greater than the tolerable UL of $2 \cdot 2 \mathrm{mg} / \mathrm{d}$.

Spearman's correlation coefficients for the estimated mean daily total dietary fluoride intakes from $3 \mathrm{~d}$ food and beverage diaries compared with the FFQ are shown in Table 3 (all statistically significant). Correlations were higher at younger ages when the diet was more limited, with the highest correlations seen at 6 months $(r=0 \cdot 90)$ and 9 months $(r=0.85)$ and a trend towards lower correlations thereafter. The lowest correlation was seen at 60 months $(r=0 \cdot 65)$.

Differences in estimated median daily dietary fluoride intake between the $3 \mathrm{~d}$ food and beverage diaries and the FFQ are summarized in Table 3. At every analysed time point, except at 12 months of age, the $3 \mathrm{~d}$ food and beverage diaries yielded higher dietary fluoride intakes than the FFQ. At 12 months, questionnaires had higher median fluoride intakes than did diaries, and this was statistically significant. At 16 months of age, diaries had higher median fluoride intakes than did questionnaires, but this was not statistically significant.

\section{Discussion}

When considering the results from this secondary data analysis, one observes that some children received substantial amounts of fluoride from dietary sources alone, not taking into consideration the amount of fluoride ingested from non-dietary sources (supplements, rinses, dental treatments, etc.), which has also been shown to be substantial $^{(17)}$. This high level of fluoride ingestion from dietary sources alone places these children at increased risk for developing dental fluorosis.

The results of this secondary data analysis suggest that an FFQ provides useful data on dietary fluoride intake compared with a $3 \mathrm{~d}$ food and beverage diary for assessing intakes from 6 to 60 months of age. Much of the dietary fluoride intake of an individual is through the consumption of beverages because of water fluoridation or use of fluoridated water to produce other beverages. A detailed FFQ can be designed to capture a significant amount of information on beverage intake with respect to nutrients of interest.

This secondary data analysis found significant, positive correlations between the dietary fluoride intakes recorded from $3 \mathrm{~d}$ food and beverage diaries and an FFQ. As the children grew older and the diet became more varied, thus potentially making it more difficult to obtain accurate dietary intake estimates, the correlations between the $3 \mathrm{~d}$ food and beverage diary and the FFQ were still fairly high and statistically significant. Although the secondary data analysis showed significant correlations, differences were observed between the estimated means as reported in the diaries and questionnaires, with an overall underestimation by the FFQ. It is possible that the estimated 
fluoride intakes as reported in the FFQ are underestimations because parents are unable to fully recall what their child ate and drank over the previous $7 \mathrm{~d}$, as opposed to the $3 \mathrm{~d}$ food and beverage diary, which is normally completed after each meal.

The correlations found in this analysis were much higher than those found by Araujo et al. ${ }^{(6)}$ and HackerThompson et al. ${ }^{(7)}$. The nutrients measured were different for each of the questionnaires, which could explain the difference in correlation coefficients. Study populations were different as well, which could also lead to differences in reported results.

Marshall et al. ${ }^{(2)}$ found that, at 6 and 12 months of age, the same FFQ yielded higher mean values for both $\mathrm{Ca}$ and vitamin $\mathrm{D}$ when compared with the $3 \mathrm{~d}$ food and beverage diary. This secondary data analysis also found that the questionnaires yielded higher mean values of dietary fluoride intake when compared with diaries at 12 months. The feeding patterns at 12 months and the design of the FFQ used in the IFS may be such that values recorded on questionnaires are higher than those from diaries. This may be due to the limited variability of the child's diet at this age and the ease of filling out the questionnaire when compared with the diary for the limited number of food categories. In addition, the assignment of fluoride values is less precise with questionnaires. It is possible that people may overestimate or forget intakes of foods and beverages when using an FFQ as they attempt to recall intake over $7 \mathrm{~d}$ compared with a food diary that is completed shortly after consumption of foods and beverages.

It is important to be cautious when interpreting these results. In absolute terms, the differences are relatively minor. For example, the statistically significant difference found between the median reported intakes from diaries and questionnaires at 12 months was $0 \cdot 01 \mathrm{mg}$ of fluoride per day (Table 3). This difference is equivalent to consuming two teaspoons of water with a fluoride level of $1.0 \mathrm{ppm}$. These differences need to be placed into context when interpreting the results from this secondary data analysis.

Another factor that needs to be considered when interpreting results from this secondary data analysis is that the fluoride database used for the IFS estimated breast milk to have a fluoride concentration of $0.01 \mathrm{ppm}$. This value is higher than most estimates of the fluoride concentration of breast milk found in other studies. Using a fluoride concentration of $0.01 \mathrm{ppm}$ for breast milk, children who consumed only breast milk might obtain $\geq 0.01 \mathrm{mg}$ of fluoride per day. This secondary data analysis defined a child as 'exclusively breast-fed' if that child was fed only breast milk and did not receive any other beverages or food, except water. In this analysis, at 6, 9 and 12 months of age, only $1 \cdot 4 \%, 0 \cdot 1 \%$ and $0 \cdot 1 \%$ of children, respectively, were exclusively breast-fed $(27 \cdot 1 \%, 18.4 \%$ and $11 \cdot 8 \%$ of children received some amount of breast milk at 6,9 and 12 months of age, respectively). It is possible that the estimated fluoride 
intake of children in this secondary data analysis who received only breast milk may be slightly higher than that found in other studies.

This secondary data analysis has several limitations. The initial study sample, although taken from eight different hospitals, was a convenience sample and not truly representative of a defined population. This cohort is a more general representation of healthy children born in those hospitals from mothers who were planning on living in the area for at least 4 years, in order to be able to track dental outcomes. On the basis of the previous parameters used to define SES categories (low SES was defined as family income $\leq$ \$US 30000 and mother not having a 4-year college degree; high SES was defined as family income $\geq \$$ US 50000 and mother having graduate or professional schooling; all other parameters were categorized as middle SES), the initial sample at recruitment was of middle/high SES (approximately $76 \%$ ) and the children who were long-term participants in the study were of even higher SES, with approximately $80 \%$ belonging to the middle and high SES categories. The study sample was predominantly white $(97 \%)$. The feeding habits of children in Iowa, as well the years of data collection, also presented possible differences from other study populations and time periods. These sample characteristics limit the generalizability of the results.

Children of low SES may show dietary patterns that are significantly different from children of higher SES, resulting in different fluoride intakes. The IFS sample was predominantly white. A study that has a sample with more racial and ethnic diversity may also present different results. It is possible that solid foods may be introduced into the diet earlier or later in populations of different racial and ethnic backgrounds. This could produce significantly different results with regard to dietary fluoride intake and correlation coefficients compared with those found in the present study. People of different ethnic backgrounds living in other countries have been shown to have very different dietary patterns compared with US children. A study sample of people from a different nation of origin living in the USA may have different patterns of dietary fluoride intake when compared with their country of origin, as well as compared with a US-born study sample. There may also be regional differences in dietary fluoride intake. Individuals in different regions of the country may consume different quantities of certain foods and beverages that could result in different dietary fluoride intake and correlation values compared with those found in the present study. Differences accruing between rural and urban settings could be important considerations as well.

The IFS consisted of children who were generally healthy. A future area of research could assess the fluoride intake of children with certain health conditions. They may present with different patterns of fluoride intake compared with a healthy population. They may also have more accurate recordings of fluoride intake, as it is more important for them to closely monitor what they eat and drink.

Another limitation that needs to be mentioned is that data were self-reported. It was not possible to validate selfreported data obtained through food and beverage diaries or through the FFQ. Moreover, both the questionnaire and the diary were mailed to the parents at the same time. In validation studies, it is generally preferable to send the 'test' tool, the questionnaire, before the standard, the diary. Because both were sent at the same time, it is possible that they were filled out simultaneously or in reverse order, resulting in an increase in agreement. Further, since the diaries included the date of completion, it is possible to have further investigated the extent to which the diaries were completed before, during and/or after the $7 \mathrm{~d}$ covered by the FFQ. Because this was not carried out, it could be seen as a limitation of this secondary data analysis. Since data were only analysed up to 60 months of age, it is important to be cautious when generalizing these results beyond this age. Sample sizes varied at the different time points because of attrition and period-specific nonresponse. The two different approaches used to collect dietary information allowed for different levels of detail. This should be kept in mind when comparing food and beverage items and amounts recorded in the food and beverage diaries and reported on the FFQ.

\section{Conclusion}

This secondary data analysis has shown that the FFQ correlates very well with $3 \mathrm{~d}$ food and beverage diaries for estimating dietary fluoride intake from 6 to 60 months of age. Higher correlations were observed at younger ages (e.g. 6 and 9 months) when the diet was limited, but even as children got older (48-60 months) and the diet became more varied diaries and questionnaires still correlated very well for dietary fluoride intake. The FFQ generally has higher degrees of compliance and is probably the tool of choice when estimating dietary fluoride intakes in large-scale longitudinal studies of fluoride intake. Further research is needed in this area to confirm these findings. Additional studies should be conducted in which the two different dietary assessment tools are mailed to study participants at separate times. Future research should also be conducted with study samples of lower SES and different racial and ethnic backgrounds to determine whether these findings hold true for different study populations.

\section{Acknowledgements}

The present study was supported in part by NIH Grants R01-DE09551, R01-DE12101 and M01-RR00059. The present paper is an original work. The authors have no 
conflict of interest to declare. S.J.R was the lead author of the paper; S.M.L., J.J.W. and J.E.G. provided content and editorial contribution; B.B. provided statistical and data quality assistance.

\section{References}

1. Subar AF (2004) Developing dietary assessment tools. J Am Diet Assoc 104, 769-770.

2. Marshall TA, Eichenberger Gilmore JM, Broffitt B et al. (2003) Relative validation of a beverage frequency questionnaire in children ages 6 months through 5 years using 3-day food and beverage diaries. J Am Diet Assoc 103, 714-720.

3. Block G, Hartman AM, Dresser CM et al. (1986) A databased approach to diet questionnaire design and testing. Am J Epidemiol 124, 453-469.

4. Burt BA \& Eklund SA (2005) Dentistry, Dental Practice, and the Community, 6th ed. St. Louis, MO: Elsevier Saunders.

5. Levy SM, Kohout FJ, Guha-Chowdhury N et al. (1995) Infants' fluoride intake from drinking water alone, and from water added to formula, beverages, and food. J Dental Res 74, 1399-1407.

6. Araujo MC, Yokoo EM \& Pereira RA (2010) Validation and calibration of a semiquantitative food frequency questionnaire designed for adolescents. J Am Diet Assoc 110, 1170-1177.

7. Hacker-Thompson A, Robertson TP \& Sellmeyer DE (2009) Validation of two food frequency questionnaires for dietary calcium assessment. J Am Diet Assoc 109, 1237-1240.
8. Institute of Medicine, Food, and Nutrition Board (1997) Dietary Reference Intakes for Calcium, Phosphorus, Magnesium, Vitamin D, and Fluoride. Washington, DC: National Academy Press.

9. Levy SM, Kiritsy MC, Slager SL et al. (1998) Patterns of dietary fluoride supplement use during infancy. J Public Health Dent 58, 228-233.

10. Levy SM, Warren JJ, Davis CS et al. (2001) Patterns of fluoride intake from birth to 36 months. J Public Health Dent 61, 70-77.

11. Warren JJ, Levy SM \& Kanellis MJ (2001) Prevalence of dental fluorosis in the primary dentition. $J$ Public Health Dent 61, 87-91.

12. Levy SM, Warren JJ \& Broffitt B (2003) Patterns of fluoride intake from 36 to 72 months of age. J Public Health Dent 63, 211-220.

13. Van Winkle SS, Levy SM, Kiritsy MC et al. (1995) Water and formula fluoride concentrations: significance for infants fed formula. Pediatr Dent 17, 305-310.

14. Kiritsy MC, Levy SM, Warren JJ et al. (1996) Assessing fluoride concentrations of juices and juice-flavored drinks. J Am Dent Assoc 127, 895-902.

15. Heilman JR, Kiritsy MC, Levy SM et al. (1997) Fluoride concentrations of infant foods. J Am Dent Assoc 128, 857-863.

16. Heilman JR, Kiritsy MC, Levy SM et al. (1999) Assessing fluoride levels of carbonated soft drinks. J Am Dent Assoc 130, 1593-1599.

17. Levy SM \& Guha-Chowdhury N (1999) Total fluoride intake and implications for dietary fluoride supplementation. J Public Health Dent 59, 211-223. 